\title{
EQUIVALENCE CLASSES OF THE 3RD GRASSMAN SPACE \\ OVER A 5-DIMENSIONAL VECTOR SPACE
}

\author{
KULDIP SINGH \\ Department of Mathematics \\ The University of New Brunswick \\ Fredericton, N.B., Canada E3B 5A3
}

(Received June 10, 1977)

ABSTRACT. An equivalence relation is defined on $\Lambda^{\mathrm{r}} \mathrm{V}$, the $\mathrm{r}^{\text {th }}$ Grassman space over $\mathrm{V}$ and the problem of the determination of the equivalence classes defined by this relation is considered. For any $r$ and $V$, the decomposable elements form an equivalence class. For $r=2$, the length of the element determines the equivalence class that it is in. Elements of the same length are equivalent, those of unequal lengths are inequivalent. When $r \geq 3$, the length is no longer a sufficient indicator, except when the length is one. Besides these general questions, the equivalence classes of $\Lambda^{3} v$, when $\operatorname{dim} \mathrm{V}=5$ are determined.

KEY WORDS AND PHRASES. Grassman space, equivalent classes, representation of equivalent classes.

AMS (MOS) SUBJECT CLASSIFICATION (1970) CODES. $14 M 15$. 
Suppose $\mathrm{V}$ is a finite dimensional vector space over an arbitrary field $F$ and $r$ is a positive integer. Consider $\Lambda^{r} v$, the rth Grassman space over $v$. We define an equivalence relation on $\Lambda^{r} V$ as follows: If $X$ and $Y$ are in $\Lambda^{r} V$, we write $X \sim Y$ iff $\exists$ a non-singular linear transformation $T: V \rightarrow V$ such that $C_{r}(T) X=Y$, where $C_{r}(T)$ is the $r$ th exterior product of $T$. Using the facts, that if $T$ and $S$ are two linear transformations of $V$, then $C_{r}(T) C_{r}(S)=C_{r}$ (TS) and if $T$ is non-singular, then $C_{r}\left(T^{-1}\right)=C_{r}(T)^{-1}$, it follows that the above relation is an equivalence relation.

We consider the problem of determining the number of equivalence classes, into which the set $\Lambda^{\mathrm{r}} \mathrm{V}$ is decomposed, along with a system of distinct representatives of these equivalence classes.

DEFINITIONS. 1. If $x \in \Lambda^{r} v$ and $x=x_{1} \wedge$. . . $\wedge x_{r}$, we say $x$ is decomposable.

2. If $X \in \Lambda^{\mathrm{r}} \mathrm{V}$, we define its length, to be denoted by $\ell(X)$ as $\ell(X)=\min \{m \mid x$ is a sum of $m$ decomposable elements of $\Lambda^{r} \mathrm{~V}$ \}.

3. If $X \in \Lambda^{r} V$, we define a subspace $[X]$ of $V$ as $[X]=n\left\{U \mid U\right.$ is a subspace of $V$ and $\left.X \varepsilon \Lambda^{r} U\right\}$.

4. If $X \in \Lambda^{\mathrm{r}} \mathrm{V}$, we define the rank of $\mathrm{X}$ to be denoted by $\rho(X)$ as $\rho(X)=\operatorname{dim}[X]$.

PROPOSITION 1. If $\mathrm{X}, \mathrm{Y} \in \Lambda^{\mathrm{r}} \mathrm{V}$ and $\mathrm{X} \sim \mathrm{Y}$, then (i) $\ell(\mathrm{X})=\ell(\mathrm{Y})$, (ii) $\mathrm{P}(\mathrm{X})=\mathrm{P}(\mathrm{Y})$.

PROOF. (i) Let $\mathrm{T}: \mathrm{V} \rightarrow \mathrm{V}$ be a n.s.1.t. such that $\mathrm{C}_{\mathrm{r}}(\mathrm{T}) \mathrm{X}=\mathrm{Y}$. If $\ell(X)=s \quad X=\sum_{i=1}^{s} x_{i}$, where $x_{i} \varepsilon \Lambda^{r_{V}}$ and $\ell\left(x_{i}\right)=1$.

Then $Y=C_{r}(T) X=\sum_{i=1}^{S} C_{r}(T) X_{i}$. This implies $\ell(Y) \leq s=\ell(X)$. Similarly $\mathrm{Y} \sim \mathrm{X}$ implies $\ell(\mathrm{Y}) \leq \ell(\mathrm{X})$ and this proves $(\mathrm{i})$. 
(ii) We first remark that if $U$ and $W$ are subspaces of $V$, then $X \in \Lambda^{r} U$ implies $\mathrm{Y} \varepsilon \Lambda^{\mathrm{r}} \mathrm{T}(\mathrm{U})$ and $\mathrm{Y} \varepsilon \Lambda^{\mathrm{r}} \mathrm{W}$ implies $\mathrm{X} \varepsilon \Lambda^{\mathrm{r}_{\mathrm{T}}} \mathrm{T}^{-1}(\mathrm{~W})$, where $\mathrm{T}: \mathrm{V} \rightarrow \mathrm{V}$ is a n.s.1.t. such that $\mathrm{Y}=\mathrm{C}_{\mathrm{Y}}(\mathrm{T}) \mathrm{X}$. From this remark, it follows easily that $[\mathrm{Y}]=\mathrm{T}[\mathrm{X}]$ and hence $\mathrm{P}(\mathrm{X})=\mathrm{P}(\mathrm{Y})$.

PROPOSITION 2. If $U$ and $W$ are subspaces of $V$, then $\Lambda^{r} U \cap \Lambda^{r} W=\Lambda^{r}(U \cap W)$. PROOF. Clearly $\Lambda^{r}(U \cap W) \subseteq\left(\Lambda^{r} U\right) \cap\left(\Lambda^{r} W\right)$. To prove the inclusion in the other direction, let $x_{1}, x_{2}, \ldots, x_{k}$ be a basis of $U \cap W$ and extend it to a basis $x_{1}, \ldots, x_{k}, y_{1}, \ldots, y_{s}$ of $U$ and a basis $x_{1}, \ldots, x_{k}, z_{1}, \ldots, z_{t}$ of $w$. Then $x_{1}, \ldots, x_{k}, y_{1}, \ldots, y_{s}, z_{1}, \ldots, z_{t}$ is a basis of $u+W$. If $A=\left\{x_{i} \wedge x_{j} \mid 1 \leq i<j \leq k\right\}, B=\left\{y_{i} \wedge y_{j} \mid 1 \leq i<j \leq s\right\}, C=\left\{z_{i} \wedge z_{j} \mid 1 \leq i<j \leq t\right\}$, $D=\left\{x_{i} \wedge y_{j} \mid 1 \leq i \leq k ; 1 \leq j \leq s\right\}, E=\left\{x_{i} \wedge z_{j} \mid 1 \leq i \leq k ; 1 \leq j \leq t\right\}$, $F=\left\{y_{i} \wedge z_{j} \mid 1 \leq i \leq s ; 1 \leq j \leq t\right\}$, then the sets $A, A \cup B \cup D, A \cup C \cup E$, and $A \cup B \cup C \cup D \cup E \cup F$ form bases of $\Lambda^{r}(U \cap W), \Lambda^{r} U, \Lambda^{r} W$ and $\Lambda^{r}(U+W)$ respectively. If $X \in\left(\Lambda^{r_{U}}\right) \cap\left(\Lambda^{r_{W}}\right)$, then $x=\sum_{A} a_{i j} x_{i} \wedge x_{j}+\sum_{B} b_{i j} y_{i} \wedge y_{j}+\sum_{D} d_{i j} x_{i} \wedge y_{j}$ and also $X=\sum_{A} a_{i j} x_{i} \wedge x_{j}+\sum_{C} c_{i j} z_{i} \wedge z_{j}+\sum_{E} e_{i j} x_{i} \wedge z_{j}$. Hence $a_{i j}=a_{i j}$ and $b_{i j}=d_{i j}=c_{i j}=e_{i j}=0$ for all the appropriate values of the indices $i$ and $j$. Thus $X \in \Lambda^{x}(U \cap W)$.

REMARK 1. The result of Proposition 2 holds for any number of subspaces of $\mathrm{V}$.

REMARK 2. If $X \in \Lambda^{r} V$ and $\boldsymbol{B}=\left\{U \mid U\right.$ is a subspace of $V, X \in \Lambda^{r} U$, then

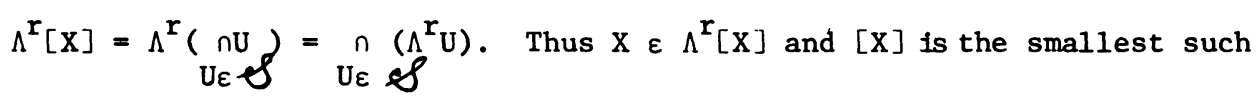
subspace of $\mathrm{V}$.

PROPOSITION 3. Let $x \in \Lambda^{2} v, \ell(x)=k$ and $x=\sum_{i=1}^{k} x_{i} \wedge y_{i}$, then $x_{1}, \ldots, x_{k}, y_{1}, \ldots, y_{k}$ are linearly independent.

PROOF. If not, then one of them (say) $y_{k}$ is a linear combination of the 
remaining $x_{1}, \ldots, x_{k}, y_{1}, \ldots, y_{k-1}$. Let $y_{k}=\sum_{i=1}^{k} a_{i} x_{i}+\sum_{j=1}^{k-1} b_{j} y_{j}$.

Then $x_{k} \wedge^{-} y_{k}=\sum_{i=1}^{k} a_{i} x_{k} \wedge x_{i}+\sum_{j=1}^{k-1} b_{j} x_{k} \wedge y_{j}$. Hence $x$ can be written as $x=\sum_{i=1}^{k-1}\left(x_{i} \wedge y_{i}+x_{k} \wedge z_{i}\right)$, where $z_{i}=a_{i} x_{i}+b_{i} y_{i}, 1 \leq 1 \leq k-1$. If $z_{i}=0$, then $\ell\left(x_{i} \wedge y_{i}+x_{k} \wedge z_{i}\right)=1$. If $z_{i} \neq 0$, let $a_{i} \neq 0$, then $x_{i} \wedge y_{i}+x_{k} \wedge z_{i}=z_{i} \wedge\left(a_{i}^{-1} y_{i}-x_{k}\right)$, thus $\ell\left(x_{i} \wedge y_{i}+x_{k} \wedge z_{i}\right) \leq 1$.

Hence $\ell(X) \leq k-1$, a contradiction.

REMARK 3. If $x \in \Lambda^{2} v, \ell(x)=k$ and $x=\sum_{i=1}^{k} x_{i} \wedge y_{i}$, then $[\mathrm{X}]=\left\langle\mathrm{x}_{1}, \ldots, \mathrm{x}_{\mathrm{k}}, \mathrm{y}_{1}, \ldots, \mathrm{y}_{\mathrm{k}}\right\rangle$.

PROOF. Let $U=\left\langle x_{1}, \ldots, x_{k}, y_{1}, \ldots, y_{k}\right\rangle$; then $[X] \subseteq U$. By Proposition 3 , $\operatorname{dim} U=2 k$. Also $X \in \Lambda^{2}[X]$; let $x=\sum_{i=1}^{k} x_{i}^{\prime} \wedge y_{i}^{\prime}, x_{i}^{\prime}, y_{i}^{\prime} \varepsilon[x], 1 \leq i \leq k$. Again by Proposition 3, $\operatorname{dim}[X] \geq 2 k$. Thus $[X]=U=\left\langle x_{1}, \ldots, x_{k}, y_{1}, \ldots, y_{k}\right\rangle$.

PROPOSITION 4. If $\mathrm{X}, \mathrm{Y} \in \Lambda^{2} \mathrm{~V}, \mathrm{P}(\mathrm{X})=\mathrm{P}(\mathrm{Y})$, then $\mathrm{X} \sim \mathrm{Y}$. PROOF. Let $\mathrm{X}=\sum_{i=1}^{k} \mathrm{x}_{i} \wedge \mathrm{y}_{i}, \mathrm{Y}=\sum_{j=1}^{s} \mathrm{x}_{j}^{\prime} \wedge \mathrm{y}_{j}^{\prime} ;$ then by Remark 3 , $[\mathrm{X}]=\left\langle\mathrm{x}_{1}, \ldots, \mathrm{x}_{\mathrm{k}}, \mathrm{y}_{1}, \ldots, \mathrm{y}_{\mathrm{k}}\right\rangle$ and $[\mathrm{Y}]=\left\langle\mathrm{x}_{1}^{\prime}, \ldots, \mathrm{x}_{\mathrm{s}}^{\prime}, \mathrm{y}_{1}^{\prime}, \ldots, \mathrm{y}_{\mathrm{s}}^{\prime}\right\rangle$. Also by Proposition 3, $P(X)=2 k, P(Y)=2 s$. Thus $k=s$. Let $T$ be a linear transformation of $\mathrm{V} \quad \mathrm{Tx}_{i}=\mathrm{x}_{i}^{\prime}, \mathrm{Ty}_{i}=\mathrm{y}_{i}^{\prime}, 1 \leq \mathrm{i} \leq \mathrm{k}$; then $\mathrm{C}_{\mathrm{r}}(\mathrm{T}) \mathrm{X}=\mathrm{Y}$. Thus $\mathrm{X} \sim \mathrm{Y}$.

PROPOSITION 5. If $x \in \Lambda^{\mathrm{r}} \mathrm{v}, \ell(\mathrm{x})=2, \mathrm{x}=\mathrm{x}_{1} \wedge \ldots \wedge \mathrm{x}_{\mathrm{r}}+\mathrm{y}_{1} \wedge \ldots \wedge \mathrm{y}_{\mathrm{r}}$, then $\mathrm{x}=\left\langle\mathrm{x}_{1}, \ldots, \mathrm{x}_{\mathrm{r}}, \mathrm{y}_{1}, \ldots, \mathrm{y}_{\mathrm{r}}\right\rangle$.

PROOF. Let $U=\left\langle x_{1}, \ldots, x_{r}, y_{1}, \ldots, y_{r}\right\rangle$; then $[X] \subseteq U$. If $[X] \neq U$, then at least one element (say) $x_{1}$ is not in $[X]$. Let $B$ be a basis of $[x]$ and extend $\{x\} \cup B$ to a basis of $U$. Let $W$ be a complement of $\left\langle x_{1}\right\rangle$ in $U$, containing $[X]$, i.e., $U=\left\langle x_{1}\right\rangle \oplus w,[X] \subseteq w$. Let $x_{i}=a_{1} x_{1}+w_{i}, 2 \leq i \leq r$ and $y_{j}=b_{j} x_{1}+w_{j}^{\prime}, 1 \leq j \leq r$, where $w_{i}, w_{j}^{\prime} \varepsilon w$. Then $x=x_{1}+x_{2}$, where $\mathrm{x}_{1} \in \mathrm{x}_{1} \wedge\left(\Lambda^{\mathrm{r}-\mathrm{l}} \mathrm{W}\right)$ and $\mathrm{x}_{2} \in \Lambda^{\mathrm{r}} \mathrm{W}$, and $\ell\left(\mathrm{x}_{\mathrm{i}}\right)=1, \mathrm{i}=1,2$. But $\mathrm{U}=\left\langle\mathrm{x}_{1}\right\rangle \oplus \mathrm{W} \Rightarrow \Lambda^{\mathrm{r}} \mathrm{U}=\mathrm{x}_{1} \wedge\left(\Lambda^{\mathrm{r}-\mathrm{l}} \mathrm{W}\right) \oplus \Lambda^{\mathrm{r}} \mathrm{W}$. Also $\mathrm{X} \varepsilon \Lambda^{\mathrm{r}}[\mathrm{X}] \subseteq \Lambda^{\mathrm{r}} \mathrm{W}$, hence 
$\mathrm{x}_{1}=\mathrm{X}-\mathrm{x}_{2} \in \Lambda^{\mathrm{r}} \mathrm{W}$. Thus $\mathrm{x}_{1}=0$ and $\mathrm{X}=\mathrm{x}_{2}=>\ell(\mathrm{X})=1$, a contradiction. Hence $[\mathrm{x}]=\mathrm{U}=\left\langle\mathrm{x}_{1}, \ldots, \mathrm{x}_{\mathrm{r}}, \mathrm{y}_{1}, \ldots, \mathrm{y}_{\mathrm{r}}\right\rangle$.

Note: The above proposition is true also for $\ell(X)=k$.

PROPOSITION 6. If $\mathrm{X}, \mathrm{Y} \varepsilon \Lambda^{\mathrm{r}} \mathrm{V}, \ell(\mathrm{X})=\ell(\mathrm{Y})=2, \mathrm{P}(\mathrm{X})=\mathrm{P}(\mathrm{Y})$, then $\mathrm{X} \sim \mathrm{Y}$.

PROOF. Let $\mathrm{x}=\mathrm{x}_{1} \wedge \ldots \wedge \mathrm{x}_{\mathrm{r}}+\mathrm{y}_{1} \wedge \ldots \wedge \mathrm{y}_{\mathrm{r}}, \mathrm{U}_{1}=\left\langle\mathrm{x}_{1}, \ldots, \mathrm{x}_{\mathrm{r}}>\right.$, $\mathrm{U}_{2}=\left\langle\mathrm{y}_{1}, \ldots, \mathrm{y}_{\mathrm{r}}\right\rangle$, then by Proposition $4,[\mathrm{x}]=\mathrm{U}_{1}+\mathrm{U}_{2}$. Let $\mathrm{z}_{1}, \ldots, \mathrm{z}_{\mathrm{k}}$ be a basis of $U_{1} \cap U_{2}$, and extend it to a basis $z_{1}, \ldots, z_{k}, u_{1}, \ldots, u_{s}$, where $\mathrm{k}+\mathrm{s}=\mathrm{r}$ of $\mathrm{U}_{1}$ and to a basis $z_{1}, \ldots, \mathrm{z}_{\mathrm{k}}, \mathrm{v}_{1}, \ldots, \mathrm{v}_{\mathrm{s}}$ of $\mathrm{U}_{2}$. Then $P(X)=k+2 s . \quad$ Since $x_{1}, \ldots, x_{r}$ and $z_{1}, \ldots, z_{k}, u_{1}, \ldots, u_{s}$ are two bases of $\mathrm{U}_{1}$, hence $\mathrm{x}_{1} \wedge \ldots \wedge \mathrm{x}_{\mathrm{r}}=a \mathrm{z}_{1} \wedge \ldots \wedge \mathrm{z}_{\mathrm{k}} \wedge \mathrm{u}_{1} \wedge \ldots \wedge \mathrm{u}_{\mathrm{s}}=\mathrm{z}_{1} \wedge \ldots \wedge \mathrm{z}_{\mathrm{k}} \wedge \overline{\mathrm{u}}_{1} \wedge \ldots \wedge \mathrm{u}_{\mathrm{s}}$, where $\overline{\mathrm{u}}_{1}=a u_{1} . \quad$ Similarly $\mathrm{y}_{1} \wedge \ldots \wedge \mathrm{y}_{\mathrm{r}}=\mathrm{bz}{ }_{1} \wedge \ldots \wedge \mathrm{z}_{\mathrm{k}} \wedge \mathrm{v}_{1} \wedge \ldots \wedge \mathrm{v}_{\mathrm{s}}=\mathrm{z}_{1} \wedge \ldots \wedge \mathrm{z}_{\mathrm{k}} \wedge \overline{\mathrm{v}}_{1} \wedge \ldots \wedge \mathrm{v}_{\mathrm{s}}$, where $\bar{v}_{1}=b v_{1}$. Hence $x=z_{1} \wedge \ldots \wedge z_{k} \wedge\left(\bar{u}_{1} \wedge u_{2} \wedge \ldots \wedge u_{s}+\bar{v}_{1} \wedge v_{2} \wedge \ldots \wedge v_{s}\right)$, where $z_{1}, \ldots, z_{k}, \bar{u}_{1}, u_{2}, \ldots, u_{s}, \bar{v}_{1}, v_{2}, \ldots, v_{s}$ is a basis of $[X]$. Similarly $\mathrm{Y}=\mathrm{z}_{1}^{\prime} \wedge \ldots \wedge \mathrm{z}_{\mathrm{k}}^{\prime} \wedge\left(\overline{\mathrm{u}}_{1}^{\prime} \wedge \mathrm{u}_{2}^{\prime} \wedge . . . \wedge \mathrm{u}_{\mathrm{s}}^{\prime}+\overline{\mathrm{v}}_{1}^{\prime} \wedge \mathrm{v}_{2}^{\prime} \wedge \ldots \wedge \mathrm{v}_{\mathrm{s}}\right)$, where $z_{1}^{\prime}, \ldots, z_{k}^{\prime}, \bar{u}_{1}^{\prime}, u_{2}^{\prime}, \ldots, u_{s}^{\prime}, \bar{v}_{1}^{\prime}, v_{2}^{\prime}, \ldots, v_{s}^{\prime}$ is a basis of $[Y]$.

Define $\mathrm{T}: \mathrm{V} \longrightarrow \mathrm{V}$, a linear transformation $\mathrm{T} \mathrm{z}_{i}=\mathrm{z}_{i}^{\prime}, \mathrm{T} \overline{\mathrm{u}}_{1}=\overline{\mathrm{u}}_{1}^{\prime}, \mathrm{Tu} \mathrm{u}_{i}=\mathrm{u}_{i}^{\prime}, \mathrm{T} \overline{\mathrm{v}}_{1}=\overline{\mathrm{v}}_{1}^{\prime}, \mathrm{T} \mathrm{v}_{i}=\mathrm{v}_{i}^{\prime}$, for $\mathrm{i}=2,3, \ldots, \mathrm{s}$. Then $\mathrm{C}_{\mathbf{r}}(\mathrm{T}) \mathrm{X}=\mathrm{Y}$; hence $\mathrm{X} \sim \mathrm{Y}$. REMARK 4. Let $X \in \Lambda^{r} V, \ell(X)=2$, then $r+1 \leq \rho(X) \leq 2 r$. PROOF. If $\mathrm{x}=\mathrm{x}_{1} \wedge \ldots \mathrm{x}_{\mathrm{r}}+\mathrm{y}_{1} \wedge \ldots \mathrm{y}_{\mathrm{r}}$, then $[\mathrm{x}]=\left\langle\mathrm{x}_{1}, \ldots, \mathrm{x}_{\mathrm{r}}, \mathrm{y}_{1}, \ldots, \mathrm{y}_{\mathrm{r}}\right\rangle$ $=\mathrm{U}_{1}+\mathrm{U}_{2}$, where $\mathrm{U}_{1}=\left\langle\mathrm{x}_{1}, \ldots, \mathrm{x}_{\mathrm{r}}\right\rangle, \mathrm{U}_{2}=\left\langle\mathrm{y}_{1}, \ldots, \mathrm{y}_{\mathrm{r}}\right\rangle, \mathrm{U}_{1} \neq \mathrm{U}_{2}$, for otherwise $\mathrm{y}_{1} \wedge \ldots \wedge \mathrm{y}_{\mathrm{r}}=\mathrm{ax}_{1} \wedge \ldots \wedge \mathrm{x}_{\mathrm{r}}$, where $\mathrm{a}$ is a scalar and $\ell(\mathrm{x})=1$. $P(X)=2 r-\operatorname{dim} U_{1} \cap U_{2}$. Hence $r+1 \leq P(X) \leq 2 r$.

THEOREM 1. Let $E(2, s)=\left\{X \mid X \varepsilon \Lambda^{r} v, \ell(X)=2, P(X)=s\right\}$, then $E(2, s), s=r+1, r+2, \ldots, 2 r$ are all the equivalence classes on the set of all vectors of $\Lambda^{r} v$, of length 2 .

PROOF. Follows from Proposition 6 and Remark 4 . 
PROPOSITION 7. Let $0 \neq \mathrm{X} \varepsilon \Lambda^{\mathrm{r}} \mathrm{V}$ and $\mathrm{x} \in \mathrm{V}$ such that $\mathrm{x} \wedge \mathrm{X}=0$; then $\mathrm{x} \varepsilon[\mathrm{X}]$.

PROOF. Let $x_{1}, x_{2}, \ldots, x_{m}$ be a basis of $[X]$. Then $\left\{\hat{x}_{\alpha} \mid \alpha \varepsilon Q_{r, m}\right\}$ is a basis of $\Lambda^{r}[X]$, where $Q_{r, m}$ is a set of all the strictly decreasing sequences of length $r$ on the integers $1,2, \ldots, m$. det $x=\sum a_{\alpha} \hat{x}_{\alpha}$;

then $x \wedge x=\sum_{\alpha} a_{\alpha} x \wedge \hat{x}_{\alpha}$. If $x \notin[x]$, then $\left\{x \wedge \hat{x}_{\alpha} \mid \alpha \varepsilon Q_{r, m}\right\}$ is a part of a basis of $\Lambda^{\mathrm{r}+1}<\mathrm{x},[\mathrm{X}]>$. Thus $\mathrm{x} \wedge \mathrm{X}=0 \Rightarrow \mathrm{a}_{\alpha}=0 \forall \alpha \varepsilon \mathrm{Q}_{\mathrm{r}, \mathrm{m}} \Rightarrow \mathrm{X}=0$, a contradiction.

PROPOSITION 8. If $0 \neq \mathrm{X} \varepsilon \Lambda^{\mathrm{r}} \mathrm{V}$ and $\mathrm{x} \notin[\mathrm{X}]$, then $[\mathrm{x} \wedge \mathrm{X}]=\langle\mathrm{x}\rangle \oplus[\mathrm{X}]$.

PROOF. By Proposition 7, $x \wedge x \neq 0$. Again by Proposition 7, since $x \wedge(x \wedge X)=0$, hence $x \in[x \wedge X]$. Clearly $[x \wedge X] \subseteq\left\langle x>\oplus[X]\right.$. Let $x, x_{1}, \ldots, x_{k}$ be a basis of $[x \wedge x]$ and extend it to a basis $x, x_{1}, \ldots, x_{k}, x_{k+1}, \ldots, x_{m}$ of $\langle\mathrm{x}\rangle \oplus[\mathrm{X}]$. If $\mathrm{U}=\left\langle\mathrm{x}_{1}, \ldots, \mathrm{x}_{\mathrm{k}}\right\rangle$, then $[\mathrm{x} \wedge \mathrm{X}]=\langle\mathrm{x}\rangle \oplus \mathrm{U}, \mathrm{U} \subseteq[\mathrm{X}]$. $\Lambda^{\mathrm{r}+1}[\mathrm{x} \wedge \mathrm{X}]=\mathrm{x} \wedge\left(\Lambda^{\mathrm{r}} \mathrm{U}\right) \oplus \Lambda^{\mathrm{r}+1} \mathrm{U}$. Let $\mathrm{x} \wedge \mathrm{X}=\mathrm{x} \wedge \mathrm{u}+\mathrm{v}$, where $\mathrm{u} \varepsilon \Lambda^{\mathrm{r}} \mathrm{U}$ and $\mathrm{v} \in \Lambda^{\mathrm{r}+1} \mathrm{U}$. Thus $\mathrm{x} \wedge \mathrm{v}=0$. If $\mathrm{v} \neq 0$, then by Proposition $7, \mathrm{x} \varepsilon[\mathrm{v}] \subset \mathrm{U}$, a contradiction. Hence $v=0$ and thus $x \wedge X=x \wedge u$. Then $x \wedge(X-u)=0$. If $\mathrm{X}-\mathrm{u} \neq 0$, then by Proposition 7, $\mathrm{x} \varepsilon[\mathrm{X}-\mathrm{u}]$. Now $\mathrm{X} \varepsilon \Lambda^{\mathrm{r}}[\mathrm{X}]$ and $\mathrm{u} \varepsilon \Lambda^{\mathrm{r}} \mathrm{U} \subseteq \Lambda^{\mathrm{r}}[\mathrm{X}]$; thus $\mathrm{X}-\mathrm{u} \varepsilon \Lambda^{\mathrm{r}}[\mathrm{X}]$. Hence $[\mathrm{X}-\mathrm{u}] \subseteq[\mathrm{X}]$. Thus $\mathrm{x} \varepsilon[\mathrm{X}-\mathrm{u}] \Rightarrow \mathrm{x} \varepsilon[\mathrm{X}]$, which is a contradiction and therefore $\mathrm{X}-\mathrm{u}=0$; i.e., $\mathrm{X}=\mathrm{u} \varepsilon \Lambda^{\mathrm{r}} \mathrm{U}$. Hence $[\mathrm{X}] \subseteq \mathrm{U}$. Also $U \subseteq[X]$, hence $U=[X]$ and $[x \wedge X]=\langle x\rangle \oplus[X]$.

PROPOSITION 9. Suppose $\mathrm{X} \varepsilon \Lambda^{2} \mathrm{~V}, \ell(\mathrm{X})=2, \mathrm{x}_{1}, \mathrm{x}_{2}$ are linearly independent vectors in $[\mathrm{X}]$. Then $\exists \mathrm{y}_{1}, \mathrm{y}_{2} \varepsilon[\mathrm{X}]$ and $\lambda \varepsilon F 3 \mathrm{x}$ has one and only one of the following representations: (i) $x=x_{1} \wedge y_{1}+x_{2} \wedge y_{2}$, (ii) $\mathrm{x}=\lambda \mathrm{x}_{1} \wedge \mathrm{x}_{2}+\mathrm{y}_{1} \wedge \mathrm{y}_{2}$.

PROOF. $X \in \Lambda^{2} V, \ell(X)=2 \Rightarrow P(X)=4$. Extend $x_{1}, x_{2}$ to a basis $x_{1}$, $x_{2}, x_{3}, x_{4}$ of $[x]$.

Then $x=\sum_{1 \leq i<j \leq 4} a_{i j} x_{i} \wedge x_{j}, a_{i j} \varepsilon F$. 
If $a_{34}=0$, take $y_{1}=a_{12} x_{2}+a_{13} x_{3}+a_{14} x_{4}$ and $y_{2}=a_{23} x_{3}+a_{24} x_{4}$, then $x=x_{1} \wedge y_{1}+x_{2} \wedge y_{2}$. If $a_{34} \neq 0$, then

$$
\left(-\lambda+a_{12}\right) a_{34}-a_{13} a_{24}+a_{14} a_{23}=0
$$

(1) has a solution in F.

Set $Y=\left(-\lambda+a_{12}\right) x_{1} \wedge x_{2}+a_{13} x_{1} \wedge x_{3}+a_{14} x_{1} \wedge x_{4}+a_{23} x_{2} \wedge x_{3}+a_{24} x_{2} \wedge x_{4}+a_{34} x_{3} \wedge x_{4} \cdot$

Then $\mathrm{Y}=-\lambda \mathrm{X}_{1} \wedge \mathrm{X}_{2}+\mathrm{X}$. Because of $(1), \ell(\mathrm{Y})=1$; also $\mathrm{Y} \varepsilon \Lambda^{2}[\mathrm{X}]$.

Thus $\mathrm{y}_{1}, \mathrm{y}_{2} \in[\mathrm{x}] \quad \mathrm{Y}=\mathrm{y}_{1} \wedge \mathrm{y}_{2}$. Hence $\mathrm{x}=\lambda \mathrm{x}_{1} \wedge \mathrm{x}_{2}+\mathrm{y}_{1} \wedge \mathrm{y}_{2}$. If $\mathrm{x}=\mathrm{x}_{1} \wedge \mathrm{y}_{1}+\mathrm{x}_{2} \wedge \mathrm{y}_{2}$ and also $\mathrm{x}=\lambda \mathrm{x}_{1} \wedge \mathrm{x}_{2}+\mathrm{z}_{1} \wedge \mathrm{z}_{2}$ then $\mathrm{x}_{1} \wedge \mathrm{x}=\mathrm{x}_{1} \wedge \mathrm{x}_{2} \wedge \mathrm{y}_{2}$ and also $x_{1} \wedge x=x_{1} \wedge z_{1} \wedge z_{2}$. Thus $0 \neq x_{1} \wedge x_{2} \wedge y_{2}=x_{1} \wedge z_{1} \wedge z_{2}$ and hence $\left\langle\mathrm{x}_{1}, \mathrm{x}_{2}, \mathrm{y}_{2}\right\rangle=\left\langle\mathrm{x}_{1}, \mathrm{z}_{1}, \mathrm{z}_{2}\right\rangle$. Let $\mathrm{z}_{1}=\mathrm{a}_{1} \mathrm{x}_{1}+\mathrm{a}_{2} \mathrm{x}_{2}+\mathrm{a}_{3} \mathrm{y}_{2}$ and $z_{2}=b_{1} x_{1}+b_{2} x_{2}+b_{3} y_{2}$. Then $z_{1} \wedge z_{2}=\left(a_{1} b_{2}-a_{2} b_{1}\right) x_{1} \wedge x_{2}+\left(a_{1} b_{3}-a_{3} b_{1}\right) x_{1} \wedge y_{2}+\left(a_{2} b_{3}-a_{3} b_{2}\right) x_{2} \wedge y_{2}$. Putting this expression for $z_{1} \wedge z_{2}$ in $x=\lambda x_{1} \wedge x_{2}+z_{1} \wedge z_{2}$, we get two different representations of $\mathrm{X}$ in the basis of $\Lambda^{2}[\mathrm{X}]$, determined by the basis $\mathrm{x}_{1}, \mathrm{x}_{2}, \mathrm{y}_{1}, \mathrm{y}_{2}$ of $[\mathrm{X}]$; thus $\mathrm{x}$ has precisely one of the two representations.

PROPOSITION 10. If $\mathrm{X}, \mathrm{Y} \in \Lambda^{\mathrm{r}} \mathrm{V}$ are decomposable, then $\mathrm{X}+\mathrm{Y}$ is decomposable iff $\operatorname{dim}[\mathrm{X}] \cap[\mathrm{Y}] \geq \mathrm{r}-1$.

PROOF. $\Leftrightarrow$ Let $X+Y$ be decomposable, and $X+Y=Z, \ell(Z) \leq 1$. Let $x=x_{1} \wedge \ldots \wedge x_{r}, Y=y_{1} \wedge \ldots \wedge y_{r}, z=z_{1} \wedge \ldots \wedge z_{r}$. If $[X]=[Z]$, then for any $i, 1 \leq i \leq r, z_{i} \wedge X=z_{i} \wedge Z=0$; but then $z_{i} \wedge Y=0$, and thus $z_{i} \varepsilon[Y]$ by Proposition 7 , and $[\mathrm{Z}]=[\mathrm{Y}]$. Hence $[\mathrm{X}]=[\mathrm{Y}]$, i.e., $\operatorname{dim}[\mathrm{X}] \cap[\mathrm{Y}]=\mathrm{r}$. If $[X] \neq[z]$, then for some $i, z_{i} \notin[X]$. But $z_{i} \wedge(X+Y)=0 \Rightarrow z_{i} \wedge X=-z_{i} \wedge Y=>\left\langle z_{i},[X]\right\rangle=\left\langle z_{i},[Y]\right\rangle$. Thus $[X],[Y]$ are $r$-dimensional subspaces in an $(r+1)$ - dim space $\left\langle z_{i},[X]\right\rangle$. Hence $\operatorname{dim}[X] \cap[Y] \geq \operatorname{dim}[X]+\operatorname{dim}[Y]-(r+1)=r-1 . \quad \Leftrightarrow$ If $\operatorname{dim}[X] \cap[Y] \geq r-1$. Let $u_{1}, \ldots, u_{r-1}$ be $1 . i$. vectors in $[X] \cap[Y]$ and extend these to a basis $\mathrm{x}, \mathrm{u}_{1}, \ldots, \mathrm{u}_{\mathrm{r}-1}$ and a basis $\mathrm{y}, \mathrm{u}_{1}, \ldots, \mathrm{u}_{\mathrm{r}-1}$ of $[\mathrm{X}]$ and $[\mathrm{Y}]$ respectively. Thus $\mathrm{X}=\mathrm{ax} \wedge \mathrm{u}_{1} \wedge \ldots \wedge u_{\mathrm{r}-1}, \mathrm{Y}=$ by $\wedge u_{1} \wedge \ldots \wedge u_{\mathrm{r}-1}$ for some $\mathrm{a}$ and $\mathrm{b}$. 
Hence $X+Y=$ (axtby) $\wedge_{1} \wedge \ldots u_{r-1}$, i.e., $X+Y$ is decomposable.

THEOREM 2. If $\operatorname{dim} V=5, X \in \Lambda^{3} \mathrm{~V}$, then $\ell(\mathrm{X}) \leq 2$.

PROOF. We shall first prove that $\ell(X) \leq 3$. Let $x_{1}, x_{2}, x_{3}, x_{4}, x_{5}$ be a basis of $v$. Then

$$
\begin{aligned}
x= & \sum_{1 \leq i<j<k \leq 5} a_{i j k} x_{i} \wedge x_{j} \wedge x_{k}=x_{1} \wedge x_{2} \wedge\left(a_{123} x_{3}+a_{124} x_{4}+a_{125} x_{5}\right) \\
& +x_{1} \wedge x_{3} \wedge\left(a_{134} x_{4}+a_{135} x_{5}\right)+x_{2} \wedge x_{3} \wedge\left(a_{234} x_{4}+a_{235} x_{5}\right) \\
& +\left(a_{145} x_{1}+a_{245} x_{2}+a_{345} x_{3}\right) x_{4} \wedge x_{5} .
\end{aligned}
$$

Let $y_{1}=a_{134} x_{4}+a_{135} x_{5}, y_{2}=a_{234} x_{4}+a_{235} x_{5}$. If $y_{1}, y_{2}$ are 1.d., then $\ell(\mathrm{X}) \leq 3$. So we assume $\mathrm{y}_{1}, \mathrm{y}_{2}$ are 1.i.; then $\left\langle\mathrm{y}_{1}, \mathrm{y}_{2}\right\rangle=\left\langle\mathrm{x}_{4}, \mathrm{x}_{5}\right\rangle$, and thus $\mathrm{x}_{4} \wedge \mathrm{x}_{5}=\lambda \mathrm{y}_{1} \wedge \mathrm{y}_{2}, \lambda \varepsilon \mathrm{F}$. Let $\mathrm{a}_{124} \mathrm{x}_{4}+\mathrm{a}_{125} \mathrm{x}_{5}=\mathrm{b}_{1} \mathrm{y}_{1}+\mathrm{b}_{2} \mathrm{y}_{2}$. Then

$$
\begin{aligned}
x= & x_{1} \wedge x_{2} \wedge\left(a_{123} x_{3}+b_{1} y_{1}+b_{2} y_{2}\right)+x_{1} \wedge x_{3} \wedge y_{1}+x_{2} \wedge x_{3} \wedge y_{2} \\
& +\lambda\left(a_{145} x_{1}+a_{245} x_{2}+a_{345} x_{3}\right) y_{1} \wedge y_{2} \\
=a_{123} x_{1} \wedge x_{2} \wedge x_{3} & +\left(x_{1}+a_{345} \lambda y_{2}\right) \wedge y_{1} \wedge\left(-b_{1} x_{2}-x_{3}+a_{145} \lambda y_{2}\right) \\
& +\left(b_{2} x_{1}-x_{3}-\left(a_{245}-a_{345} b_{1}\right) \lambda y_{1}\right) \wedge x_{2} \wedge y_{2} .
\end{aligned}
$$

Hence $\ell(X) \leq 3$.

Let $\mathrm{x}=\mathrm{x}_{1}+\mathrm{x}_{2}+\mathrm{x}_{3}$, where $\mathrm{x}_{1}, \mathrm{x}_{2}, \mathrm{x}_{3}$ are decomposable, $\mathrm{x}_{1}=\mathrm{x}_{1} \wedge \mathrm{x}_{2} \wedge \mathrm{x}_{3}$, $\mathrm{x}_{2}=\mathrm{y}_{1} \wedge \mathrm{y}_{2} \wedge \mathrm{y}_{3}, \mathrm{x}_{3}=\mathrm{z}_{1}{ }^{\wedge z_{2} \wedge \mathrm{z}_{3}}$. Then $1 \leq \operatorname{dim}\left[\mathrm{x}_{1}\right] \cap\left[\mathrm{x}_{2}\right] \leq 3$.

CASE 1. $\operatorname{dim}\left[\mathrm{X}_{1}\right] \cap\left[\mathrm{X}_{2}\right]=3$. Then $\mathrm{X}_{2}=\lambda \mathrm{X}_{1}$ for some $\lambda$ and thus $\ell(\mathrm{X}) \leq 2$.

$\operatorname{CASE} 2$. $\operatorname{dim}\left[\mathrm{x}_{1}\right] \cap\left[\mathrm{x}_{2}\right]=2$. Let $\mathrm{u}_{1}, \mathrm{u}_{2}, v$ and $\mathrm{u}_{1}, \mathrm{u}_{2}, \mathrm{w}$ be bases of $\left[\mathrm{x}_{1}\right]$ and $\left[x_{2}\right]$ respectively. Then $x_{1}=\lambda u_{1} \wedge u_{2} \wedge v$ and $x_{2}=\lambda u_{1} \wedge u_{2} \wedge w$. Then $\ell(X) \leq 2$.

$\operatorname{CASE}$ 3. $\operatorname{dim}\left[\mathrm{x}_{1}\right] \cap\left[\mathrm{x}_{2}\right]=1$. $\operatorname{det} \mathrm{u}_{1}, \mathrm{u}_{2}, \mathrm{u}_{3}$ and $\mathrm{u}_{1}, \mathrm{u}_{4}, \mathrm{u}_{5}$ be bases of $\left[x_{1}\right]$ and $\left[x_{2}\right]$ respectively. Then $x_{1}=u_{1} \wedge u_{2} \wedge u_{3}, x_{2}=u_{1} \wedge u_{4} \wedge u_{5}$; we have assumed the co-effs. to be absorbed with the vectors $u_{i}$ 's and $v_{i}$ 's. Then $\mathrm{X}_{1}+\mathrm{X}_{2}=\mathrm{u}_{1} \wedge \mathrm{Y}$, where $\mathrm{Y}=\mathrm{u}_{2} \wedge \mathrm{u}_{3}+\mathrm{u}_{4}^{\wedge \mathrm{u}_{5}}$. Also $\left[\mathrm{X}_{1}\right]+\left[\mathrm{X}_{2}\right]=\mathrm{V}$. 
Since $\operatorname{dim}<u_{2}, u_{3}, u_{4}, u_{5}>\cap\left[x_{3}\right] \geq 2$, we can take $x_{3}=w_{1} \wedge w_{2} \wedge w_{3}$, where $\mathrm{w}_{1}, \mathrm{w}_{2} \varepsilon<\mathrm{u}_{2}, \mathrm{u}_{3}, \mathrm{u}_{4}, \mathrm{u}_{5}>$. By Proposition 9, $\mathrm{v}_{1}, \mathrm{v}_{2}$ and $\lambda \quad \mathrm{Y}=\lambda \mathrm{w}_{1} \wedge \mathrm{w}_{2}+\mathrm{v}_{1} \wedge \mathrm{v}_{2}$ or $\mathrm{Y}=\mathrm{w}_{1} \wedge \mathrm{v}_{1}+\mathrm{w}_{2} \wedge \mathrm{v}_{2}$. If $\mathrm{Y}=\lambda \mathrm{w}_{1} \wedge \mathrm{w}_{2}+\mathrm{v}_{1} \wedge \mathrm{v}_{2}$, then $\mathrm{X}=\mathrm{u}_{1} \wedge \mathrm{Y}+\mathrm{w}_{1} \wedge \mathrm{w}_{2} \wedge \mathrm{w}_{3}$ has length $\leq 2$. If $\mathrm{Y}=\mathrm{w}_{1} \wedge \mathrm{v}_{1}+\mathrm{w}_{2} \wedge \mathrm{v}_{2}$, then since $\mathrm{u}_{1}, \mathrm{w}_{1}, \mathrm{w}_{2}, \mathrm{v}_{1}, \mathrm{v}_{2}$ is also a basis of $v$, let $w_{3}=a_{1} u_{1}+a_{2} w_{1}+a_{3} w_{2}+a_{4} v_{1}+a_{5} v_{2}$. Then $\mathrm{x}=\mathrm{x}_{1}+\mathrm{x}_{2}+\mathrm{x}_{3}=\left(\mathrm{u}_{1}-\mathrm{a}_{4} \mathrm{w}_{2}\right) \wedge \mathrm{w}_{1} \wedge \mathrm{v}_{1}+\mathrm{u}_{1} \wedge \mathrm{w}_{2} \wedge \mathrm{v}_{2}+\left(\mathrm{a}_{5} \mathrm{v}_{2}+\mathrm{a}_{1} \mathrm{u}_{1}\right) \wedge \mathrm{w}_{1} \wedge \mathrm{w}_{2}$ has length $\leq 2$, since $\mathrm{z}=\mathrm{u}_{1} \wedge \mathrm{w}_{2} \wedge \mathrm{v}_{2}+\left(\mathrm{a}_{5} \mathrm{v}_{2}+\mathrm{a}_{1} \mathrm{u}_{1}\right) \wedge \mathrm{w}_{1} \wedge \mathrm{w}_{2}$ and $\left.\operatorname{dim}<u_{1}, w_{2}, v_{2}\right\rangle n\left\langle a_{5} v_{2}+a_{1} u_{1}, w_{1}, w_{2}\right\rangle \geq 2$ implies $\ell(Z) \leq 1$.

REMARK. There exists $X \in \Lambda^{3} v$ with $\ell(X)=2$; for if $x_{1}, x_{2}, x_{3}, x_{4}, x_{5}$ is a basis of $V$ and $x=x_{1} \wedge x_{2} \wedge x_{3}+x_{1} \wedge x_{4} \wedge x_{5}$, then $\ell(X)=2$, by Proposition 10 .

REMARK. If $X \in \Lambda^{3} \mathrm{~V}, \operatorname{dim} V=5, \ell(X)=2$, then $P(X)=5$; for let $\mathrm{x}=\mathrm{x}_{1}+\mathrm{x}_{2}$, where $\ell\left(\mathrm{x}_{1}\right)=\ell\left(\mathrm{x}_{2}\right)=1$. Since $\mathrm{x}$ is not decomposable, then by Proposition 10, $\operatorname{dim}\left[\mathrm{x}_{1}\right] \cap\left[\mathrm{x}_{2}\right]<2$ and hence $\operatorname{dim}[X]>\operatorname{dim}\left[X_{1}\right]+\operatorname{dim}\left[x_{2}\right]-\operatorname{dim}\left[x_{1}\right] \cap\left[X_{2}\right]=4$, i.e., $P(X)=5$.

It follows from Proposition 6 that if $\mathrm{X}, \mathrm{Y} \varepsilon \Lambda^{3} \mathrm{~V}$ and $\ell(\mathrm{X})=\ell(\mathrm{Y})$, then $\mathrm{X} \sim \mathrm{Y}$. Hence all the equivalence classes of $\Lambda^{3} \mathrm{~V}$ are given by

$$
\begin{aligned}
& \mathrm{S}_{0}=\left\{\mathrm{X} \mid \mathrm{X} \in \Lambda^{3} \mathrm{v}, \ell(\mathrm{X})=0\right\}=\{0\} \\
& \mathrm{S}_{1}=\left\{\mathrm{X} \mid \mathrm{X} \in \Lambda^{3} \mathrm{v}, \ell(\mathrm{X})=1\right\} \\
& \mathrm{S}_{2}=\left\{\mathrm{X} \mid \mathrm{X} \in \Lambda^{3} \mathrm{v}, \ell(\mathrm{X})=2\right\} .
\end{aligned}
$$

ACKNOWLEDGMENT. The author is grateful to Professor R. Westwick for his invaluable help in the preparation of this manuscript.

\section{REFERENCES}

1. Hodge, W. V. D. and D. Pedoe. Methods of Algebraic Geometry, Vol. 1, University Press, Cambridge, 1953.

2. Lim, M. J. S. L-2 Subspaces of Grassmann Product Spaces, Pacific J. Math. 33 (1970) 167-182.

3. Gurewich, G. B. Foundations of the Theory of Algebraic Invariants, P. Noordhoff Ltd., Groningen, The Netherlands, 1964. 


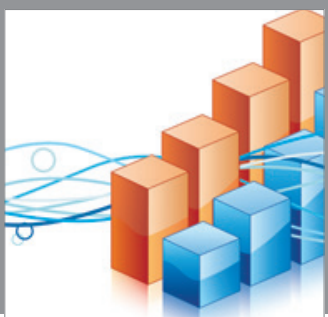

Advances in

Operations Research

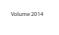

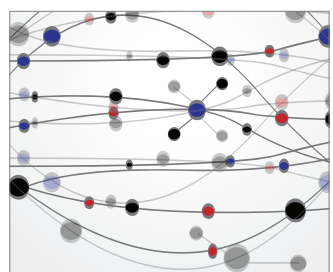

\section{The Scientific} World Journal
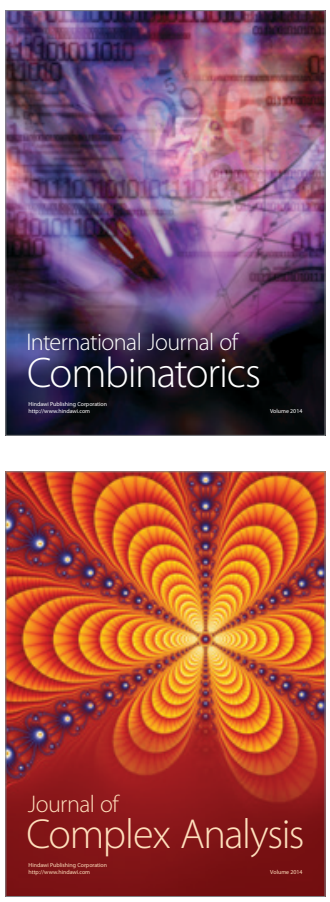

International Journal of

Mathematics and

Mathematical

Sciences
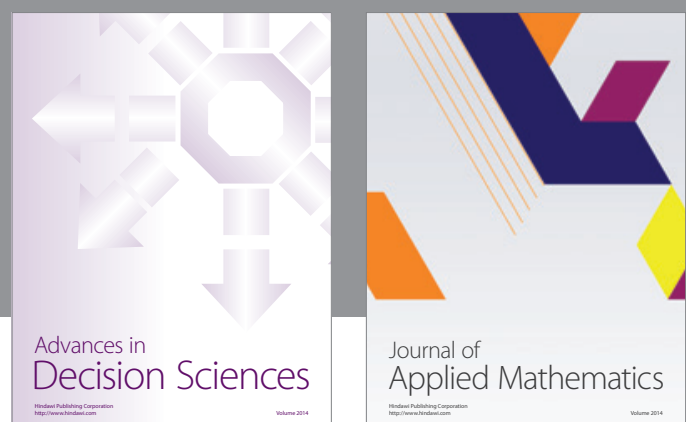

Journal of

Applied Mathematics
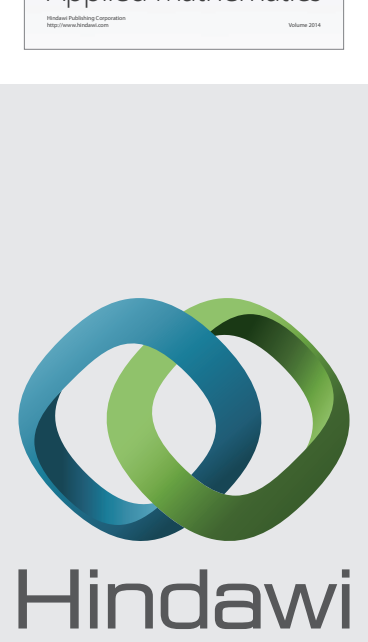

Submit your manuscripts at http://www.hindawi.com
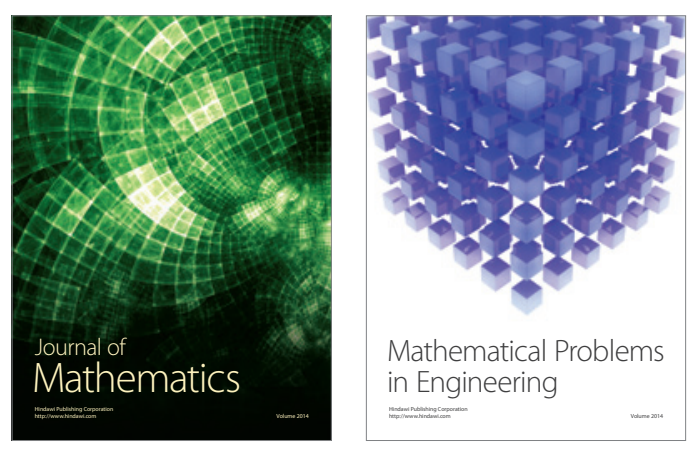

Mathematical Problems in Engineering
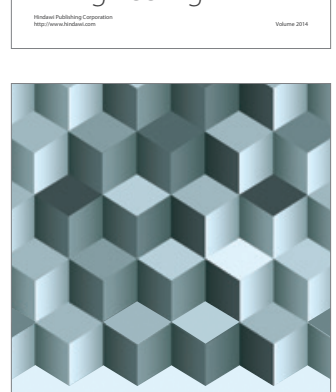

Journal of

Function Spaces
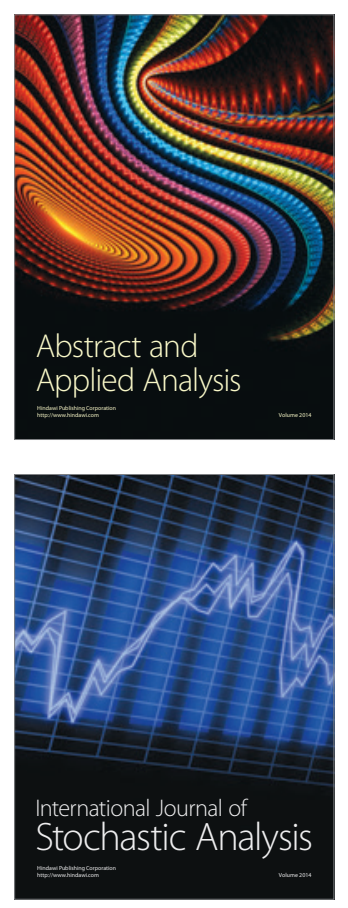

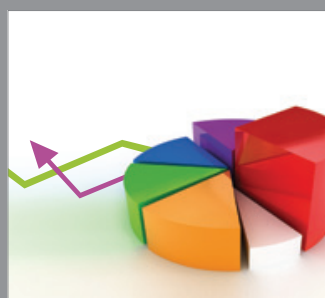

ournal of

Probability and Statistics

Promensencen
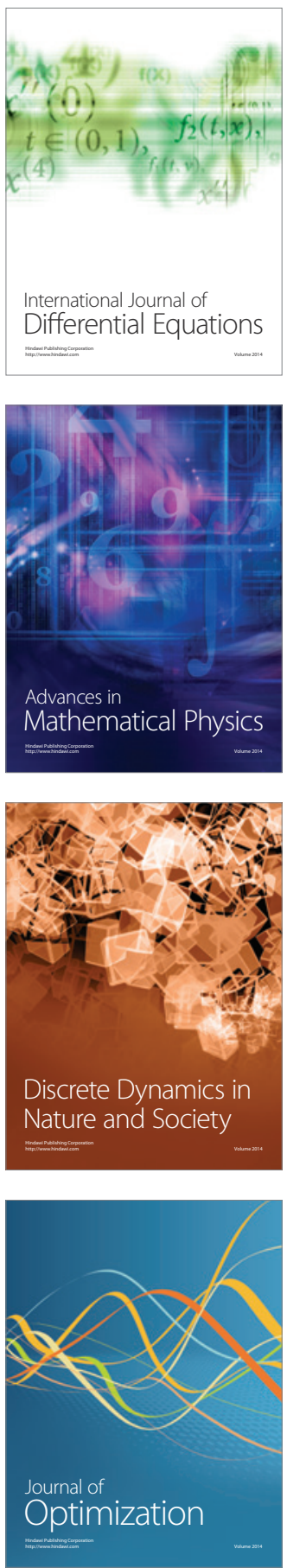